\title{
The Outcome of Inpatient Acute Febrile Illness in A Referral Tropical Health Center in Nepal
}

\author{
Vivek Kattel $^{1 *}$, Yamuna Agrawal ${ }^{2}$, Naveen Kumar Pandey ${ }^{3}$, Semanta Dahal ${ }^{4}$, Basudha Khanal ${ }^{5}$ \\ 1 Tropical and Infectious Disease Unit, BPKIHS, Dharan, Nepal; ${ }^{2}$ Department of Pathology, BPKIHS, Dharan, Nepal; ${ }^{3}$ \\ Department of Cardiology, BPKIHS, Dharan, Nepal; ${ }^{4}$ Department of Anesthesia, Institute of Medicine, Kathmandu, Nepal; ${ }^{5}$ \\ Department of Microbiology, BPKIHS, Dharan, Nepal
}

\begin{abstract}
Introduction: Acute Febrile Illness (AFI) is a common clinical syndrome presenting at tropical health centers. The challenges in resource-limited set up are an undifferentiated clinical manifestation with wide differentials and inadequate laboratory diagnostic support. With this background, we conducted a study to look at the outcome of AFI spectrum presented at BP Koirala Institute of Health Sciences (BPKIHS), a referral medical school hospital in eastern Nepal.

Objective: To determine the etiological diagnosis and hospital-based outcome of AFI.

Methods: It was a prospective observational study of the AFI inpatient cases in the Department of Internal Medicine from $1^{\text {st }}$ January 2013 to $31^{\text {st }}$ December 2013. Considering a $15 \%$ prevalence of acute febrile in patients with a $95 \%$ confidence interval and $95 \%$ power of study a sample size of 196 was calculated. Assuming 25\% as a sampling error 245 patients were enrolled. The patient was diagnosed and treated as per the hospital protocol developed by Tropical and Infectious Disease Unit. Case record form was used to record and tabulated in an excel sheet. Descriptive and analytic statistics were used.

Results: The incidence of AFI was 12\% (557) among the 4669 inpatient cases. Among 245 enrolled cases, 61\% presented as localized fever. The most common clinical diagnosis was pneumonia (29\%), urinary tract infection (18\%), meningitis (11\%) and tropical disease (14\%) that includes malaria, dengue, rickettsia, and leptospirosis. The etiological diagnosis was established among 26\% (64). Among AFI cases sepsis, acute renal injury and septic shock was present in $18 \%, 11 \%$, and $6 \%$ respectively. The putative diagnosis could not be made in $18 \%$ (44) of the cases and they were treated empirically with dual antibiotics (injectable $3^{\text {rd }}$ generation cephalosporin with macrolides or fluoroquinolones or aminoglycosides). Favorable outcomes in term of clinical cure were seen in $76 \%$ (186) of the cases.

Conclusion: Establishment of etiological diagnosis is logistically not feasible in developing the world. Contextual guidelines for undifferentiated fever may be a possible option for improving the outcome of undifferentiated fever in Nepal.
\end{abstract}

Keywords: Acute febrile illness; Nepal; Outcomes; Undifferentiated fever

\section{INTRODUCTION}

Of all the manifestations of infections, fever is the most common and most constant presentation by the human host.
Undifferentiated fever is one of the challenges for diagnostic workup to doctors. Sentinel hospital-based studies, performed over a defined period of time, have proven useful to gather

Corresponding author: Vivek Kattel, Tropical and Infectious Disease Unit, BPKIHS, Dharan, Nepal, E-mail: vivekkattel@bpkihs.edu

Received date: July 11, 2019; Accepted date: July 27, 2019; Published date: August 06, 2019

Citation: Kattel V, Agrawal Y, Pandey NK, Dahal S, Khanal B (2019) Outcome OF Inpatient Acute Febrile Illness in A Referral Tropical Health Center in Nepal. J Trop Dis 7:328.

Copyright: (C) 2019 Kattel V, et al. This is an open-access article distributed under the terms of the Creative Commons Attribution License, which permits unrestricted use, distribution and reproduction in any medium, provided the original author and source are credited. 
clinical and public health information in countries that lack resources for long term routine diagnostic technique [1]. Many laboratory setups are designed to diagnose the burden of endemic infectious disease with substantial importance. Hence the other emerging and reemerging febrile illness remain undefined unless there is an outbreak with significant morbidity and mortality burden [2]. A most common cause of the acute febrile illness is self-limiting viral fever however these patients are unlikely to be admitted as an inpatient in public referral centers where bed occupancy is almost $100 \%$. Thus with this background, we intended to study the epidemiology and outcome of acute undifferentiated patients.

\section{OBJECTIVES}

To determine the etiologies and hospital-based outcome of inpatient acute febrile illness cases.

\section{METHODOLOGY}

It was a prospective observational study conducted from January 1 to December 31, 2013, conducted at BPKIHS. BPKIHS is a medical university with a 1000 bedded referral hospital. It receives patients from hills, mountain, and tropics of eastern Nepal and adjoining border area of Bihar, India. Considering a $15 \%$ prevalence of acute febrile inpatients with a $95 \%$ confidence interval and $95 \%$ power of study a sample size of 196 were calculated. Assuming $25 \%$ as a sampling error 245 patients were enrolled. Adult ( $>14$ years) with fever for a duration of fewer than 2 weeks were included in the study. All healthcareassociated infections, traumatic infections, iatrogenic infections and noninfectious febrile cases like underlying autoimmune diseases or drug-induced fever were excluded. Patients with uncontrolled noncommunicable diseases like diabetes mellitus, chronic obstructive pulmonary disease, chronic liver disease, and chronic kidney disease were also excluded. The patient was diagnosed and treated as per the hospital protocol developed by Tropical and Infectious Disease Unit. The information regarding the patients was recorded in pilot-tested case record form maintaining patient confidentiality. At the end of the assessment, each case was classified as confirmatory diagnosed or clinically diagnosed or underdiagnosed. Confirmatory diagnosed was defined when an organism is established and that explains the illness. Clinically diagnosed as defined when history, examination and supportive laboratory test findings were coherent and contributory to the diagnosis. The undiagnosed case was defined either any cases that did not fit in confirmatory or clinical diagnosis or expired before diagnostic workup could. Hospital-based outcomes were measured as favorable for the clinical cure and unfavorable for the any among the referred or Left Against Medical Advised (LAMA) or expired cases. For descriptive statistics, percentage, proportion, ratio, mean, median, standard deviation are applied. For inferential statistics, chi-square and t-test are applied to find out the significant differences between clinical features, examination finding, and laboratory findings.

\section{RESULTS}

There were 4669 inpatients admitted in the year 2013 out of which $12 \%$ (557) were an acute febrile illness. The average hospital stay of this AFI patient was 7.2 days (SD 2.3) whereas the average stay of overall inpatient was 5.7 days (SD 3.1) (pvalue<0.0001). The burden of febrile patients was maximum during the hot and humid climate (Figure 1).

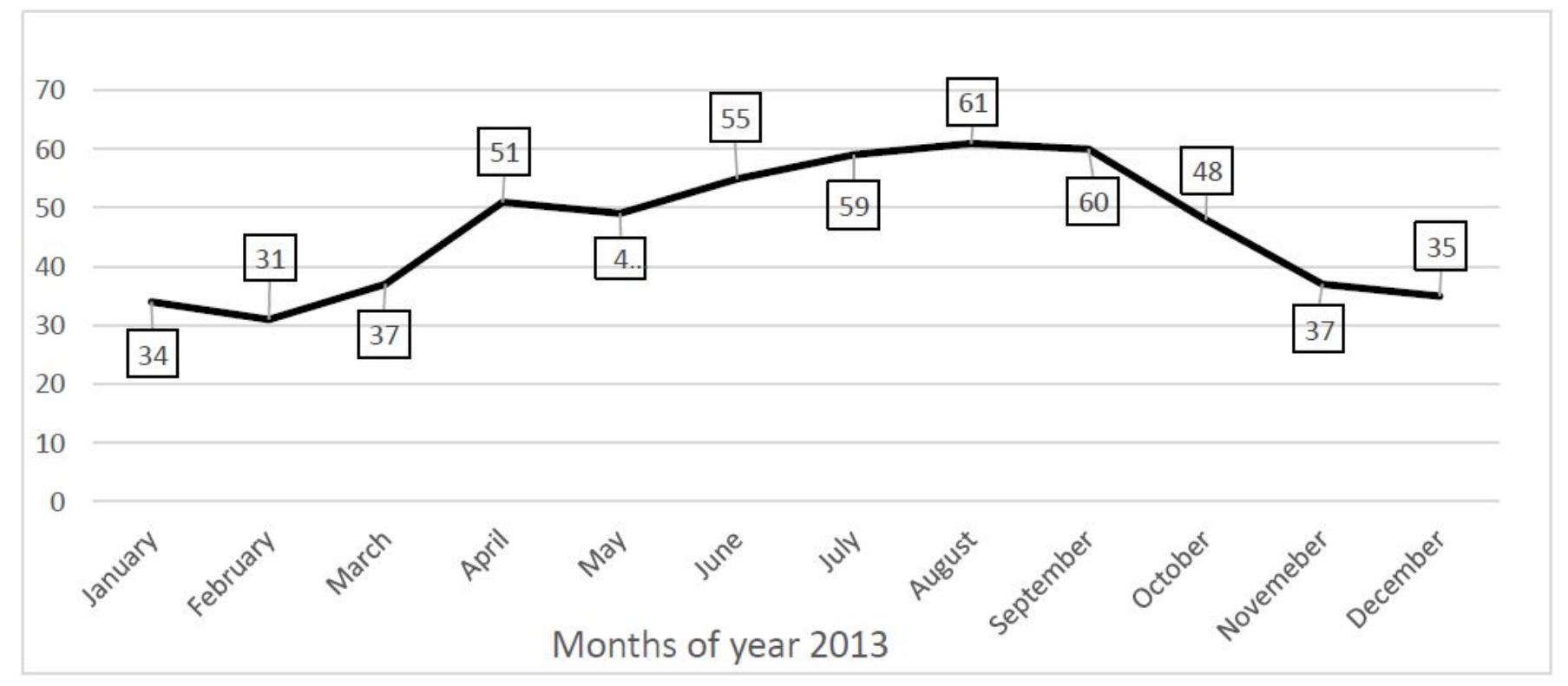

Figure 1: Trend of AFI inpatient over the year 2013.

Among the 245 enrolled cases mean and the median age was 37 years (SD 11.2) and 47 years respectively. The male to female ratio was 1.2 . Among 245 cases $76 \%$ (186) were from a tropical climate, 91\% (223) had exposure to frequent mosquito bites, $68 \%$ (167) had exposure to vegetation, $41 \%$ (100) had overcrowding and 39\% (96) had poor access to the safe water supply. Self or pharmacy prescribed antibiotics exposure was observed in 58\% (142) most commonly oral azithromycin in $39 \%$ (96) cases, oral aminopenicillin or oral cefixime in $24 \%$ (58) cases and oral fluoroquinolones (ofloxacin and 
levofloxacin) in $15 \%$ (37) cases. The common symptoms after the fever were cough (60\%), severe headache (49\%) and burning micturition (44\%) (Table 1).

Table 1: Percentage of positive clinical symptoms and signs $(n=245)$.

\begin{tabular}{|c|c|c|}
\hline Symptoms & Number & Percentage \\
\hline Cough & 147 & $60 \%$ \\
\hline Headache & 121 & $49 \%$ \\
\hline Burning micturition & 108 & $44 \%$ \\
\hline Loose motion & 45 & $18 \%$ \\
\hline Altered sensorial & 39 & $16 \%$ \\
\hline Vomiting & 22 & $9 \%$ \\
\hline Joint pain & 19 & $8 \%$ \\
\hline Rashes & 14 & $6 \%$ \\
\hline Fits and convulsion & 8 & $3 \%$ \\
\hline $\begin{array}{l}\text { Auscultation suggestive of } \\
\text { Pneumonia (Localized } \\
\text { crepitations and/or bronchial } \\
\text { breath) }\end{array}$ & 92 & $38 \%$ \\
\hline $\begin{array}{l}\text { Sign suggestive of Meningitis } \\
\text { GCS < } 12 \text { and/or positive } \\
\text { Kernicks and/or Brudzinski }\end{array}$ & 86 & $35 \%$ \\
\hline $\begin{array}{l}\text { Signs suggestive of UTI } \\
\text { suprapubic and/or renal } \\
\text { angle tenderness }\end{array}$ & 67 & $27 \%$ \\
\hline Icterus & 7 & 35 \\
\hline
\end{tabular}

Localizing symptoms or signs were present among 61\% (149) of the cases. Alarming vital signs in terms of any one of the followings GCS less than 12, tachycardia (pulse rate more than $100 / \mathrm{min}$ ), tachypnea (respiratory rate more than $24 / \mathrm{min}$ ) and systolic blood pressure less than $90 \mathrm{mmHg}$ was found in $66 \%$ (162) patients at admission. Red flag signs like severe pallor, moderate to severe icterus, cyanosis, diffuse rashes, generalized tender lymphadenopathy, clinically moderate to severe per abdominal organomegaly, $\mathrm{SpO}_{2}$ less than $90 \%$ despite supplement oxygen and delirium was found in 32\% (78) cases (Table 2).

\begin{tabular}{lll}
\hline Characteristics & Yes & No \\
\hline Undifferentiated fever & $96(39 \%)$ & $149(61 \%)$ \\
\hline Stable vitals & $83(34 \%)$ & $162(67 \%)$ \\
\hline
\end{tabular}

\begin{tabular}{lll}
\hline Red flags signs & $167(68 \%)$ & $78(32 \%)$ \\
\hline Sepsis & $44(18 \%)$ & $201(82 \%)$ \\
\hline AKI & $27(11 \%)$ & $218(89 \%)$ \\
\hline Septic shock & $15(6 \%)$ & $230(94 \%)$ \\
\hline
\end{tabular}

Table 2: Characteristics of inpatient acute febrile illness $(n=245)$.

The most common clinical diagnosis was pneumonia (71, 29\%) followed by urinary tract infections $(44,18 \%)$, meningitis (27, $11 \%)$ and tropical diseases like malaria, dengue, leptospirosis, and rickettsia $(34,14 \%)$. Seventy-three percent of the rickettsia was reported post-monsoon (August, September, and October) and all of them had exposure to vegetation. Eschar was present if $64 \%(7 / 11)$ of rickettsia. All cases of dengue gave a history of the outbreak of fever in their community. Total leukocyte counts were normal among $89 \%(16 / 18)$ of dengue and rickettsia. Twenty four malarial patients (96\%) were afebrile within 24 hours of injectable Artesunate and $72 \%(8 / 11)$ of rickettsia was afebrile within 48 hours of doxycycline treatment. Meningitis was statistically associated with unfavorable outcome $(p=0.03)$. The probable diagnosis could not be made in $18 \%$ (45) of cases and labeled as Pyrexia Of Unknown Origin (PUO) (Table 3).

Table 3: Clinical diagnosis and outcome $(n=245)$.

\begin{tabular}{llll}
\hline Clinical diagnosis & $\begin{array}{l}\text { Favorable } \\
\text { outcome }\end{array}$ & $\begin{array}{l}\text { Unfavorable } \\
\text { outcome }\end{array}$ & p-value \\
\hline Pneumonia (n=71) & $52(73 \%)$ & $19(27 \%)$ & 0.46 \\
\hline $\begin{array}{l}\text { Urinary tract } \\
\text { infections (n=44) }\end{array}$ & $38(86 \%)$ & $6(14 \%)$ & 0.08 \\
\hline Meningitis (n=27) & $17(63 \%)$ & $10(37 \%)$ & 0.03 \\
\hline Malaria (n=12) & $12(100 \%)$ & 0 & 0.3 \\
\hline Rickettsia (n=11) & $7(64 \%)$ & $4(36 \%)$ & 0.9 \\
\hline Dengue (n=7) & $7(100 \%)$ & 0 & 0.7 \\
\hline Leptospirosis $(n=4)$ & $3(75 \%)$ & $1(25 \%)$ & 0.3 \\
\hline Others (n=24) & $19(79 \%)$ & $5(21 \%)$ & $13(29 \%)$ \\
\hline PUO (n=45) & $32(71 \%)$ & $58(24 \%)$ & \\
\hline Total & $187(76 \%)$ & & 0.7 \\
\hline
\end{tabular}

The proportion of unfavorable outcomes in terms of Left Against Medical Advice (LAMA), mortality and referred cases were 7\% (18), 9\% (22) and 7\% (18) respectively (Figure 2). 


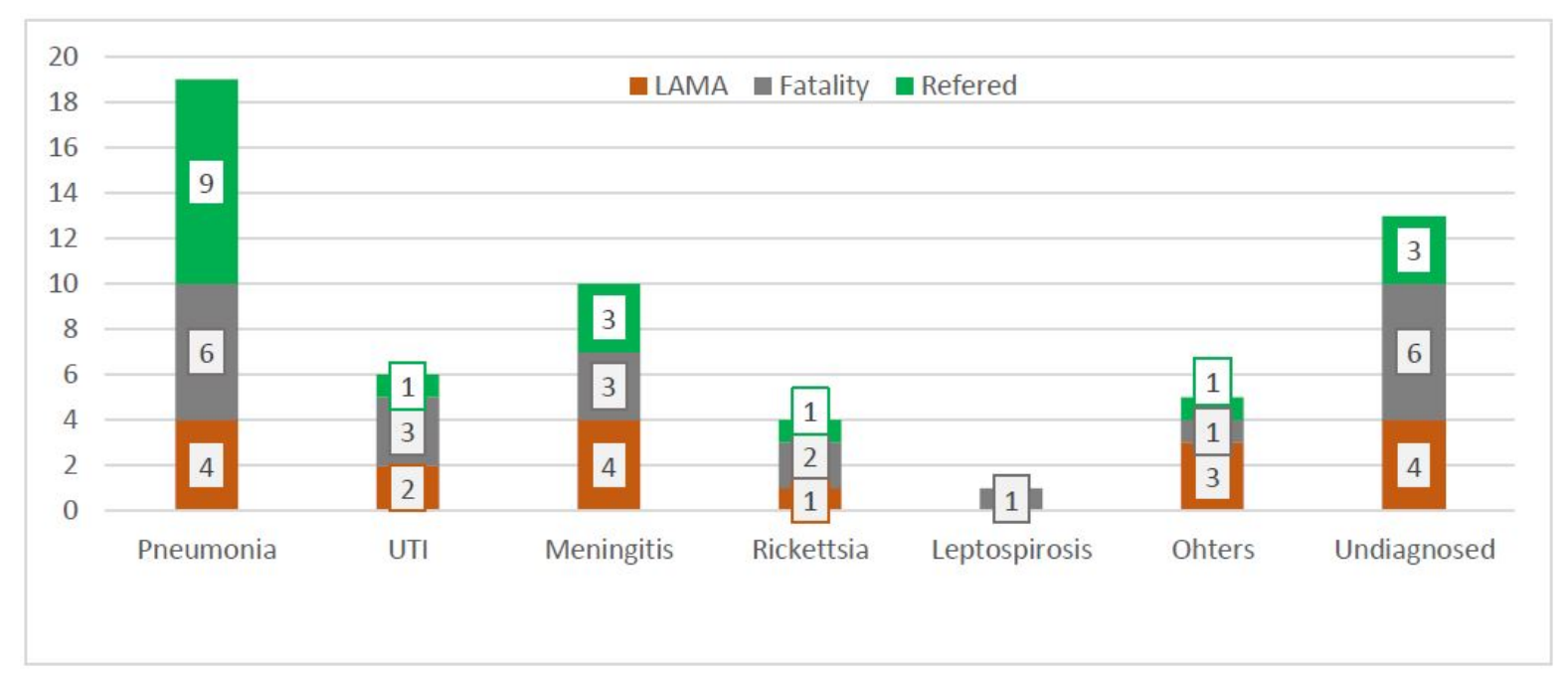

Figure 2: Proportion of unfavorable outcome.

Preference to doxycycline, fluoroquinolones, and aminoglycosides were based upon the history of antibiotics consumption, cost, and likelihood of possible adverse drug reaction. Favorable outcome was observed in $85 \%$ of the doxycycline group, 68\% of aminoglycosides group and $60 \%$ of fluoroquinolones group. Among the 17 undiagnosed cohorts, a therapeutic trial of two standard doses of injection Artesunate 12 hours apart was successful in 9 cases. Response with injection Artesunate was seen within 24 hours of treatment among these nine cases (Table 4).

Table 4: Characteristics of undiagnosed fever (PUO).

\begin{tabular}{lll}
\hline Clinical diagnosis & $\begin{array}{l}\text { Favorable } \\
\text { outcome }\end{array}$ & $\begin{array}{l}\text { Unfavorable p-value } \\
\text { outcome }\end{array}$
\end{tabular}

(32) (13)

\begin{tabular}{llll}
\hline Mean Age & $41(\mathrm{SD} 6.8)$ & $39(\mathrm{SD} 6.1)$ & 0.36 \\
\hline Sepsis (N=14) & 8 & 6 & 0.17 \\
\hline AKI (N=6) & 3 & 3 & 0.22 \\
\hline Septic shock (N=7) & 2 & 5 & 0.007 \\
\hline $\begin{array}{l}\text { Doxycycline group (N=13) } \\
\text { Fluroquinolones } \\
\text { group (N=22) }\end{array}$ & 11 & 2 & 0.20 \\
\hline $\begin{array}{l}\text { Aminoglycosides } \\
\text { group (N=10) }\end{array}$ & 6 & 7 & 0.67 \\
\hline $\begin{array}{l}\text { Artesunate group (N=17) } \\
\text { (10) }\end{array}$ & 9 & 4 & 0.65 \\
\hline
\end{tabular}

Body fluid conventional culture and demonstration of antigen by Rapid Diagnostic Test (RDT) was the available test for etiological diagnosis. Among the 245 cases, the etiological diagnosis was established among 26\% (64) of cases (Table 5). Blood culture was positive among $8.6 \%$ (14/163) cases with Salmonella growth of 2 samples. Urine culture was positive among $28.7 \%$ (31/108) cases most common E. coli. In our study, the RDT kit available for dengue and malaria had a sensitivity of $71 \%(5 / 7)$ and $67 \%(8 / 12)$ respectively. Pneumonia was more of clinical diagnosis with the missed rate of statistical significance $(p=0.0003)$.

Table 5: Etiological diagnosis among AFI.

\begin{tabular}{lll}
\hline Clinical Diagnosis & $\begin{array}{l}\text { Confirmatory } \\
\text { Diagnosis }\end{array}$ & Missed rate \\
\hline Pneumonia $(\mathrm{n}=71)$ & 10 & $86 \%(\mathrm{p}=0.0003)$ \\
\hline $\begin{array}{l}\text { Urinary tract infections } \\
(\mathrm{n}=44)\end{array}$ & 17 & $61 \%(\mathrm{p}=0.37)$ \\
\hline Meningitis $(\mathrm{n}=27)$ & 12 & $56 \%(\mathrm{p}=0.21)$ \\
\hline Malaria $(\mathrm{n}=12)$ & 10 & $17 \%(\mathrm{p}=0.0003)$ \\
\hline Rickettsia $(\mathrm{n}=11)$ & 4 & $64 \%(\mathrm{p}=0.78)$ \\
\hline Dengue $(\mathrm{n}=7)$ & 5 & $29 \%(\mathrm{p}=0.031)$ \\
\hline Leptospirosis $(\mathrm{n}=4)$ & 1 & $75 \%(\mathrm{p}=0.76)$ \\
\hline Others $(\mathrm{n}=24)$ & 5 & $79 \%(\mathrm{p}=0.27)$ \\
\hline Total $(\mathrm{n}=200)$ & 64 & $68 \%$ \\
\hline
\end{tabular}

\section{DISCUSSION}

This is the first study to systematically examine the infectious causes of acute febrile illness among adult patients in eastern Nepal. This study has measured the diagnostic capability and treatment outcome of the tropical and infectious disease center. The clinical and etiological diagnostic yield of the center for acute febrile illness is $82 \%$ and $26 \%$ respectively. A study carried by David R. Murdoch et al. during the year 2001 at Patan Hospital in Kathmandu [2] among 876 consecutive febrile adults had putative pathogens identifications in 323 (37\%) patients. 
The etiological diagnosis of acute febrile illness varies between $8 \%-80 \%$ in different studies carried out in Asia depending upon resource setting and epidemiological factors [3]. Blood culture growth was found in $8.6 \%$ in our study. A study done at Kathmandu in the year 2007 found 28\% positive blood culture reports with most common growth being Salmonella species [4]. The reason for low blood culture yield could be a higher rate of exposure to antibiotics (58\%) before blood sampling in our study. The favorable treatment outcome in our study was $76 \%$. Among these febrile cases, $61 \%$ had localizing signs and symptoms which guided us for starting appropriate antimicrobial agents. However, in the absence of localization of infections broad-spectrum antibiotics and additional antimicrobial agents based upon endemic infections is a rationale choice. Most of the local guidelines for febrile illness are based upon the epidemiological data and endemic disease. The most common differentials of undifferentiated fever in Nepal is enteric fever [5]. This could be the reason for the use of trial of azithromycin, cefixime, and quinolones among generalized fever cases at primary consultation. Our study contradicts with the common differential of nonspecific fever as a vector-borne disease like malaria, rickettsia, leptospirosis, and dengue. In a cohort study of 1258 patients in South India, scrub typhus was the most common cause of acute undifferentiated febrile illness (35.9\%) followed by dengue (30.6\%), malaria (10.4\%), enteric fever (3.7\%), and leptospirosis (0.6\%) [6]. Scrub typhus and dengue were the most common cause of fever in Central India [7]. The national guideline may consider this fact and advocate primary physician for considering these differentials besides enteric fever and treat.

The sensitivity of malaria Rapid Diagnostic Test (RDT) in our study was $67 \%$ despite the sensitivity of the kit was to detect a minimum of 200 parasites per $\mathrm{mL}$ of blood. Malarial RDT is likely to yield false negative in situations where patient are exposed to antimalarial drugs or antibiotics like sulpha drugs, clindamycin, and doxycycline that has antimalarial activities. Poor sensitivity of RDT in clinical practices may be the reason for the low threshold to give antimalarial at clinics especially at rural settings [8]. This might be a barrier for the elimination target for malaria in South Asia. In such a scenario, Polymerase Chain Reaction (PCR) can be valuable but only in limited setup. In our study, the therapeutic response of injectable antimalarial was observed by 24 hours. For very sick undifferentiated fever patients, two doses of injectable antimalarial may prove to be cheaper than PCR, especially in resource-limited setup. A specific guideline for antimalarial in negative RDT or microscopy is warranted in the absence of PCR facilities.

In the absence of diagnostic facilities for these common differentials, epidemiological data can be a clue. Warm and humid climate with exposure to vegetation, toxic look, Escher, normal leukocyte counts and thrombocytopenia is a great diagnostic clue for rickettsia $[9,10]$. Besides therapeutic response to doxycycline against Rickettsia by 48 hours could be a diagnostic clue in absence confirmatory test. Febrile illness outbreak at clusters in a small geographic area can be an epidemiological clue for dengue fever. Lack of specific epidemiological determinants to describe the etiology of fever is one of the challenges to develop national guidelines in Nepal.
Despite meticulous workup, fever becomes undiagnosed for prolong duration which is termed as Pyrexia of Unknown Origin (PUO). PUO remains a great diagnostic and therapeutic challenges in clinical practices. In our study $18 \%$ of cases were PUO. The treatment success rate was $71 \%$ among PUO. Among PUO cases septic shock was significantly associated with unfavorable outcomes in our study. PUO is usually caused by common disorders, often with an unusual presentation [11]. The treatment success rate of PUO by ceftriaxone and doxycycline arm was $85 \%$ followed by $68 \%$ on ceftriaxone and aminoglycosides arm and $60 \%$ on ceftriaxone and fluoroquinolones arm. Twenty percentage of PUO life was saved by two doses trail of injectable antimalarial agents followed by oral antimalarial in our study. Management of febrile illness in Nepal is a challenging task due to knowledge gaps and lack of expensive diagnostic facilities [12]. High yield laboratory is a success story for PUO however cost remains the main barrier for these modalities in developing countries. Hence in circumstances with a lack of highly sensitive and specific test a therapeutic diagnostic trial may be demanded. Single-day trail of injectable antimalarial and two days trail of doxycycline can be lifesaving, time-saving and cost-effective modalities especially among very sick PUO patients or undifferentiated fever in tropics.

Besides diagnostic yield, the challenge of acute fever is to distinguish the threatening from the trivial one [13]. We found $66 \%$ of febrile cases were hemodynamically unstable at presentations and $32 \%$ had extra warning signs. The higher burden of unfavorable outcome was found in meningitis, rickettsia, PUO, and pneumonia. Meningitis was significantly associated with death and LAMA in our study. Poor clinical outcomes were reported among undiagnosed cases, nervous system involvement and acute respiratory distress patients in even taken care at ICU [14].

\section{CONCLUSION}

Acute febrile illness is challenging if present as multisystem involvements, hemodynamically unstable and undiagnosed despite meticulous workup. Availability of better diagnostic facility is a theoretical answer for undifferentiated fever however cost remain the major limitations. Guideline based upon the clinical characterization and the therapeutic outcome can be a cost-effective modality in resource-limited setup.

\section{ACKNOWLEDGEMENT}

We would like to acknowledge the staffs of tropical and infectious disease unit.

\section{LIMITATION OF THE STUDY}

Single centered study.

\section{CONFLICT OF INTEREST}

The author declares no conflict of interest. 


\section{REFERENCES}

1. Archibald LK, Reller LB. Clinical microbiology in developing country. Emerg Infect Dis. 2001;7:302-305.

2. Murdoch DR, Woods CW, Zimmerman MD, Dull PM, Belbase RH, Keenan AJ, et al. The etiology of febrile illness in adults presenting to Patan hospital in Kathmandu, Nepal. Am J Trop Med Hyg. 2004;70:670-675.

3. Susilawati TN, McBride WJH. Acute undifferentiated fever in Asia: A review of the literature. Southeast Asian J Trop Med Public Health. 2014;45:719-726.

4. Blacksell SD, Sharma NP, Phumratanaprapin W, Jenjaroen K, Peacock SJ, White NJ, et al. Serological and blood culture investigations of Nepalese fever patients. Trans R Soc Trop Med Hyg. 2007;101:686-690.

5. Andrews JR, Vaidya K, Bern C, Tamrakar D, Wen S, Madhup S, et al. High rates of enteric fever diagnosis and lower burden of culture-confirmed disease in peri-urban and rural Nepal. J Infect Dis. 2018;10:S214-S221.

6. Morch K, Manoharan A, Chandy S, Chacko N, Alvarez-Uria G, Patil S, et al. Acute undifferentiated fever in India: a multicentre study of aetiology and diagnostic accuracy. BMC Infect Dis. 2017; 17:665.

7. Shelke YP, Deotale VS, Maraskolhe DL. Spectrum of infections in acute febrile illness in central India. Indian J Med Microbiol. 2017;35:480-484.
8. Joshi R, Colford JM, Jr. Reingold AL, Kalantri S. Nonmalarial acute undifferentiated fever in a rural hospital in central India: diagnostic uncertainty and overtreatment with antimalarial agents. The American journal of tropical medicine and hygiene. 2008;78:393-399.

9. Aung AK, Spelman DW, Murray RJ, Graves S. Rickettsial infections in Southeast Asia: implications for local populace and febrile returned travelers. Am J Trop Med Hyg. 2014;91:451-460.

10. Shapiro MR, Fritz CL, Tait K, Paddock CD, Nicholson WL, Abramowicz KF, et al. Rickettsia: a newly recognized cause of eschar-associated illness in California. Clin Infect Dis. 2010;50:541-548.

11. Schneider T, Loddenkemper C, Rudwaleit M, Lode H, Zeitz M. Fever of unknown origin in the 21st century: infectious diseases. Dtsch Med Wochenschr. 2005;130:2708.

12. Koirala DK, Chappuis F, Verdonck K, Rijal S, Boelaert M. Persistent febrile illnesses in Nepal: A systematic review. Indian J Med Res. 2018;148:385-395.

13. Crump JA, Kirk MD. Estimating the burden of febrile illnesses. PLoS Negl Trop Dis. 2015;9:e0004040.

14. Muthaiah B, Thippeswamy T, Kondareddy S, ChIkkegowda P. Study of Aetiology and outcome in acute febrile illness patients with multiple organ dysfunction syndrome. J Clin Diagn Res. 2016;10:16-18. 